\title{
Intensity-modulated radiotherapy for stage I glottic cancer: a short-term outcomes compared with three-dimensional conformal radiotherapy
}

\author{
Ick Joon Cho', Woong-Ki Chung ${ }^{1}$, Joon Kyoo Lee², Min-Cheol Lee ${ }^{3}$, Jayeong Paek', \\ Yong-Hyub Kim', Jae-Uk Jeong ${ }^{1}$, Mee Sun Yoon', Ju-Young Song ${ }^{1}$, Taek-Keun Nam', \\ Sung-Ja Ahn', Dong Hoon Lee ${ }^{2}$, Tae Mi Yoon², Sang-Chul Lim² \\ 'Department of Radiation Oncology, Chonnam National University Hwasun Hospital, Chonnam National University Medical \\ School, Hwasun, Korea \\ ${ }^{2}$ Department of Otorhinolaryngology-Head and Neck Surgery, Chonnam National University Hwasun Hospital, Chonnam National \\ University Medical School, Hwasun, Korea \\ ${ }^{3}$ Department of Pathology, Chonnam National University Hwasun Hospital, Chonnam National University Medical School, \\ Hwasun, Korea \\ ${ }^{4}$ Institute for Biomedical Science, Chonnam National University Hwasun Hospital, Hwasun, Korea
}

Purpose: To investigate the differences in treatment outcomes between two radiation techniques, intensity-modulated radiotherapy (IMRT) and three-dimensional conformal radiotherapy (3DCRT).

Materials and Methods: We retrospectively analyzed $160($ IMRT $=23,3 D C R T=137)$ patients with stage I glottic cancer treated from January 2005 through December 2016. The IMRT was performed with TomoTherapy (16 patients), volumetric-modulated arc therapy (6 patients), and step-and-shoot technique (1 patient), respectively. The 3DCRT was performed with bilateral parallel opposing fields. The median follow-up duration was 30 months (range, 31 to 42 months) in the IMRT group and 65 months (range, 20 to 143 months) in the 3DCRT group.

Results: The 5-year overall survival and 3-year local control rates of the 160 patients were $95.7 \%$ and $91.4 \%$, respectively. There was no significant difference in 3 -year local control rates between the IMRT and 3DCRT groups (94.4\% vs. 91.0\%; $p=0.587)$. Thirteen of 137 patients in the 3DCRT group had recurrences. In the IMRT group, one patient had a recurrence at the true vocal cord. Patients treated with IMRT had less grade 2 skin reaction than the 3DCRT group, but this had no statistical significance ( $4.3 \%$ vs. $21.2 \% ; p=0.080)$. Conclusion: IMRT had comparable outcomes with 3DCRT, and a trend of less acute skin reaction in stage I glottic cancer patients

Keywords: Glottis, Laryngeal neoplasms, Intensity-modulated radiotherapy, Outcomes

Introduction
Early glottic cancer is successfully treated with definitive surgery or radiotherapy. In a systemic review of T1 glottic

Received 20 May 2019, Revised 22 September 2019, Accepted 07 October 2019.

Correspondence: Woong-Ki Chung, Department of Radiation Oncology, Chonnam National University Hwasun Hospital, Chonnam National University Medical School, 322 Seoyang-ro, Hwasun 58128, Korea. Tel: +82-61-379-7200, Fax: +82-61379-7249, E-mail: wkchung@jnu.ac.kr (http://orcid.org/0000-0001-6049-3263)

(C) This is an Open Access article distributed under the terms of the Creative Commons Attribution Non-Commercial License (http://creativecommons.org/ licenses/by-nc/4.0/) which permits unrestricted non-commercial use, distribution, and reproduction in any medium, provided the original work is properly cited.

www.e-roj.org 
Table 1. Patient and tumor characteristics by radiotherapy techniques

\begin{tabular}{|c|c|c|c|c|}
\hline Characteristic & $\begin{array}{c}\text { Total } \\
(\mathrm{n}=160)\end{array}$ & $\begin{array}{c}\text { 3DCRT } \\
(n=137)\end{array}$ & $\begin{array}{c}\text { IMRT } \\
(n=23)\end{array}$ & $p$-value ${ }^{a)}$ \\
\hline Age (yr) & $64(35-84)$ & $65(35-84)$ & $62(43-78)$ & 0.108 \\
\hline Sex & & & & 1.000 \\
\hline Male & $155(96.9)$ & $133(97.1)$ & $22(95.7)$ & \\
\hline Female & $5(3.1)$ & $4(2.9)$ & $1(4.3)$ & \\
\hline Differentiation & & & & 0.164 \\
\hline Well & $113(70.6)$ & $96(70.1)$ & $17(73.9)$ & \\
\hline Moderate & $30(18.8)$ & $25(18.2)$ & $5(21.7)$ & \\
\hline Poor & $1(0.6)$ & $0(0)$ & $1(4.3)$ & \\
\hline No inform & $16(10.0)$ & $16(11.7)$ & $0(0)$ & \\
\hline T stage & & & & 0.786 \\
\hline T1a & $126(78.8)$ & 107 (78.1) & 19 (82.6) & \\
\hline T1b & $34(21.3)$ & $30(21.9)$ & $4(17.4)$ & \\
\hline A-com invasion & & & & 0.070 \\
\hline No & $118(73.8)$ & 105 (76.6) & $13(56.5)$ & \\
\hline Yes & $42(26.3)$ & $32(23.4)$ & $10(43.5)$ & \\
\hline RT dose prescribed (Gy) & $66.0(60.0-66.6)$ & $66.0(60.0-66.0)$ & $66.0(66.0-66.0)$ & 0.578 \\
\hline RT duration (day) & $48(39-66)$ & $48(39-66)$ & $49(45-54)$ & 0.203 \\
\hline Follow-up duration (mo) & $59(20-143)$ & $65(20-143)$ & $30(31-42)$ & $<0.001$ \\
\hline
\end{tabular}

Values are presented as median (range) or number (\%).

IMRT, intensity-modulated radiotherapy; 3DCRT, three-dimensional conformal radiotherapy; RT, radiotherapy.

${ }^{a}$ The chi-square test, Mann-Whitney U-test, and Student t-test were used to test differences between the two treatment groups.

cancer, there was no significant difference between transoral laser microsurgery and radiotherapy regarding local control [1].

With definitive radiotherapy, more than 90\% local control and larynx preservation has been achieved for $\mathrm{T1}$ glottic cancer without serious late toxicity [2-5]. The choice of treatment method is based on the patient's wishes, surgeon's experience, and institutional factors [6].

Three-dimensional conformal radiotherapy (3DCRT) has been the most widely used irradiation technique for T1-T2 tumors. Recently, the intensity-modulated radiotherapy (IMRT) technique has been widely applied for the treatment of headand-neck cancers, but there is controversy over its use for early glottic cancer [7]. The objective of this study is to report the short-term clinical outcomes of IMRT in T1 glottic cancer patients compared with 3DCRT.

\section{Materials and Methods}

\section{Patients}

From January 2005 through December 2016, 160 consecutive patients with $\mathrm{T} 1 \mathrm{glottic}$ cancer were treated with 3DCRT
(137 patients) or IMRT (23 patients) at our hospital. All the patients were histologically confirmed as having squamous cell carcinomas. Patients who had a history of cancer before radiotherapy were excluded. For staging work-ups, all the patients had a physical examination, laryngeal microsurgery (LMS) with biopsy, laboratory examination, and computed tomography (CT) of the head, neck and chest region. The staging was performed according to the 6th edition (2002) or 7th edition (2010) of the TNM classification of American Joint Committee on Cancer (AJCC), which did not differ for T1 glottic cancer. The patients were followed weekly during radiation treatment to check acute toxicity. Acute and late complications during or after radiotherapy were recorded according to Radiation Therapy Oncology Group (RTOG) criteria [8]. After the completion of treatment, the patients were seen in an outpatient clinic every 2 to 3 months for 3 years, and then at 6-month intervals after the fourth year. Patient characteristics are summarized in Table 1. This study was approved by the Institutional Review Board of Chonnam National University Hwasun Hospital (No. CNUHH-2017-157). 


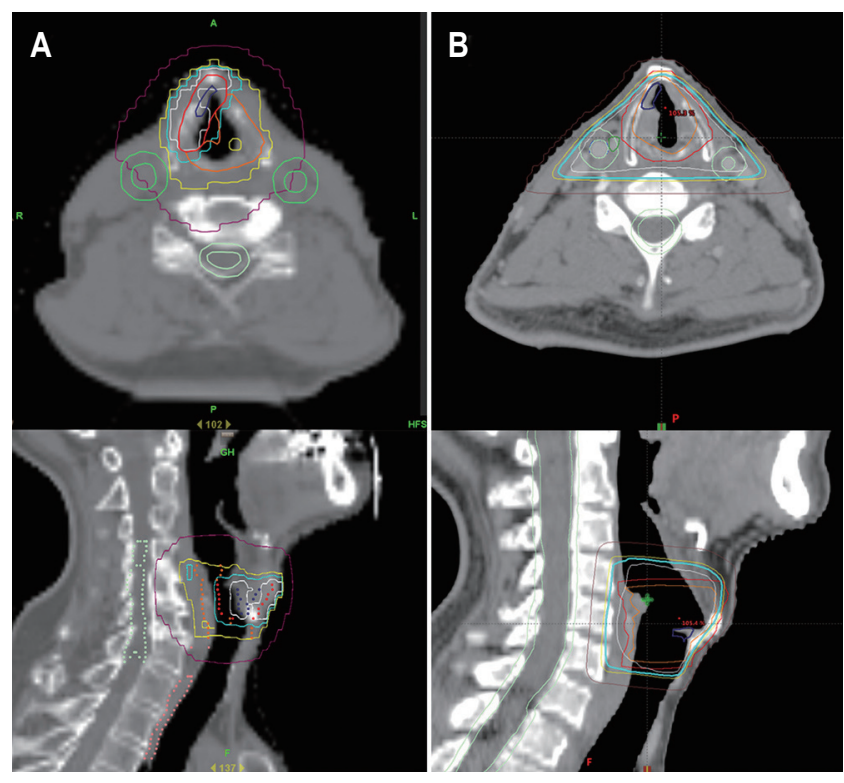

Fig. 1. Planning target volume (red line) and volume received 95\% of prescribed dose (cyan line) of patient who received intensity-modulated radiotherapy (A) and three-dimensional conformal radiotherapy (B).

\section{Target volume definition}

During the setup of the radiation-treated patients, all patients were fixed on a couch using a 5-point thermoplastic mask and neck support and CT scans were made with a $2.5-\mathrm{mm}$ slice thickness in their supine position.

For the IMRT, we included the involved glottic lesion, ipsilateral true vocal cord (TVC), and anterior commissure in the clinical target volume (CTV-1). The potential high-risk area of local recurrence (CTV-2) covered both the true and false vocal cords, subglottis and part of the supraglottis; CTV2 extended to the thyroid notch cranially, cricoid cartilage inferiorly, thyroid cartilage anteriorly and laterally, and arytenoid cartilage posteriorly. For the 3DCRT, the CTV included both the true and false vocal cords, subglottis and part of the supraglottis. To generate a planning target volume (PTV), a 3to 5 - mm margin was added to the CTV (Fig. 1).

The organs at risk included the thyroid gland and spinal cord. The carotid arteries were not routinely delineated. We retrospectively delineated carotid arteries as organs at risk for the reporting following the recommendation of the literature [9]. The right and left carotid arteries were delineated up to 2 $\mathrm{cm}$ superiorly and inferiorly beyond PTV-2, and the volumes were expanded by $4 \mathrm{~mm}$ to create planning organ at risk volume (PRV).

\section{Radiotherapy planning and delivery}

Dose prescription for the IMRT group was set to the following: $66 \mathrm{~Gy}$ in 33 fractions to the PTV-1 and $59.4 \mathrm{~Gy}$ in 33 fractions to the PTV-2. We prescribed dose constraints to normal tissue as follows: a maximum of 45 Gy for the spinal cord, maximum of $54 \mathrm{~Gy}$ for the esophagus, and mean dose of less than $30 \mathrm{~Gy}$ for the thyroid glands. Dose constraints on the carotid arteries were imposed for 2 patients in the IMRT group $(n=23)$. For the IMRT, two parameters were used to achieve planning goals: $95 \%$ of PTV-1 or PTV-2 should reach more than the 95\% isodose level of the prescribed dose, and the maximum point dose of them should be lower than 110\% of the prescribed dose. Dose plans were evaluated in terms of physical quantities based on cumulative dose-volume histograms, dose statistics and isodose curves. The Eclipse planning system (Varian Medical System, Palo Alto, CA, USA) was used for the linear accelerator-based IMRT. Helical TomoTherapy (TomoTherapy Inc., Madison, WI, USA) was performed on 16 patients. For the IMRT by helical TomoTherapy we used a field width of $2.5 \mathrm{~cm}$, a modulation factor of 2.468 , and a pitch of 0.287 . All the patients had image-guided radiation therapy (IGRT) with daily cone beam CT. Volumetric modulated arc therapy (VMAT) with Novalis TX (Varian Medical System) with $2.5 \mathrm{~mm}$ multileaf collimators (daily IGRT; cone beam CT, six-directional couch) was performed on six patients. VMAT treatment plans used a one arc (5 patients) or double arc (1 patient) therapy approach. One arc of the VMAT plan used a $320^{\circ}$ gantry rotation from $200^{\circ}$ to $160^{\circ}$ for clockwise (CW) rotation. The two-arc plan used $360^{\circ}$ rotation from $270^{\circ}$ to $90^{\circ}$ for $\mathrm{CW}$, and $90^{\circ}$ to $270^{\circ}$ for counter-clockwise (CCW) rotation, respectively. The collimator angle for $\mathrm{CW}$ or CCW gantry rotation was $30^{\circ}$ or $330^{\circ}$. The IMRT by step-and-shoot delivery technique of a linear accelerator with an On-Board Imager (Varian Medical Systems) was performed on one patient. For this technique, seven beam angles of $110^{\circ}, 70^{\circ}, 40^{\circ}, 0^{\circ}, 320^{\circ}, 290^{\circ}$, and $260^{\circ}$ were preselected on the PTV, and all the collimator angles were $0^{\circ}$. For the 3DCRT, we used two parallel opposing fields with or without wedges. The bolus was not applied. The field borders are as follows: superiorly under the hyoid bone, inferiorly it covered cricoid cartilage, anteriorly it covered $1 \mathrm{~cm}$ outside the skin, and the posterior border was on the anterior one third of the vertebral body. Patients were treated with a 6-MV X-ray from a linear accelerator.

The conformity and homogeneity indices for all IMRT plans were calculated using the following formula according to the recommendation of ICRU report 83 [10]. 
Table 2. Physical parameters by radiotherapy technique

\begin{tabular}{|c|c|c|c|}
\hline & IMRT $(n=21)^{a)}$ & 3DCRT $(n=137)$ & $p$-value \\
\hline$V_{95}$ & $35.69 \pm 14.69$ & $90.54 \pm 30.67$ & $<0.001$ \\
\hline$D_{2}$ & $68.64 \pm 1.20$ & $70.50 \pm 1.10$ & \\
\hline$D_{50}$ & $67.11 \pm 0.86$ & $68.90 \pm 1.10$ & \\
\hline$D_{98}$ & $65.64 \pm 0.80$ & $64.07 \pm 2.10$ & \\
\hline Homogeneity index (\%) & $4.47 \pm 0.95$ & $8.90 \pm 3.90$ & 0.002 \\
\hline Conformity index & $2.33 \pm 1.04$ & $4.21 \pm 2.98$ & 0.006 \\
\hline \multicolumn{4}{|l|}{ OAR } \\
\hline Spinal cord, Dmax & $32.22 \pm 5.29$ & $5.12 \pm 4.18$ & $<0.001$ \\
\hline Both thyroid, mean & $15.13 \pm 5.67$ & $14.98 \pm 6.39$ & 0.919 \\
\hline \multicolumn{4}{|l|}{ Carotid artery ${ }^{\mathrm{b})}$} \\
\hline \multicolumn{4}{|l|}{ Ipsilateral } \\
\hline$V_{35}$ & $23.68 \pm 17.19$ & $8.56 \pm 2.09$ & $<0.001$ \\
\hline$V_{50}$ & $8.02 \pm 7.71$ & $7.73 \pm 1.98$ & 0.810 \\
\hline \multicolumn{4}{|l|}{ Contralateral } \\
\hline$V_{35}$ & $17.61 \pm 16.77$ & $8.57 \pm 2.12$ & 0.001 \\
\hline$V_{50}$ & $4.43 \pm 6.75$ & $7.69 \pm 2.04$ & 0.004 \\
\hline
\end{tabular}

Values are presented as mean \pm standard deviation.

IMRT, intensity-modulated radiotherapy; 3DCRT, three-dimensional conformal radiotherapy; $V_{95}$, volume received $95 \%$ of prescribed dose; OAR, organ at risk; PTV, planning target volume; $D_{x \prime}$ minimum dose received $x \%$ of PTV.

Conformity index $=\frac{V_{95}}{\text { PTV }}$.

Homogeneity index $(\%)=\frac{D_{2}-D_{98}}{D} \times 100$.

${ }^{a}$ Two patients with carotid dose constraint were excluded.

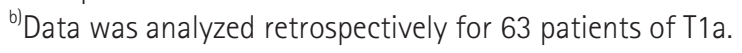

$$
\text { Conformity index }=\frac{V_{95}}{\text { PTV }}
$$

$V_{95}$ is defined as the volume irradiated more than $95 \%$ of the prescription dose.

$$
\text { Homogeneity index }(\%)=\frac{D_{2}-D_{98}}{D_{50}} \times 100
$$

$D_{2}, D_{981}$ and $D_{50}$ were defined as the minimum doses delivered to $2 \%, 98 \%$ and $50 \%$ of PTV.

\section{Statistical analysis}

The primary end points of this study were local control and overall survival rates. The treatment outcomes of IMRT were compared with the 3DCRT group. The time of follow-up was based on the date of completion of radiation treatment. The duration of survival was measured from the date of the end of radiotherapy until the date of death from any cause or last follow-up. The duration of local recurrence-free survival was measured to the date of the first recurrence of glottic lesion. The chi-square test, Mann-Whitney U-test, and Student t-test were used to test differences between the two treatment groups. The Kaplan-Meier method for the survival rate and the log-rank test were performed to identify prognostic factors. Statistical analysis was performed using SPSS version 23 (IBM Corp., Armonk, NY, USA). $p<0.05$ was considered to be statistically significant.

\section{Results}

\section{Patient characteristics}

There were no significant differences in the distribution of age, sex, degree of differentiation, T-stage, anterior commissure invasion, irradiation dose, and duration of radiotherapy between the IMRT and 3DCRT groups. The median followup duration of the IMRT group was shorter than that of the 3DCRT group (30 vs. 65 months; $p<0.001$ ) (Table 1).

The treated volume of 95\% of prescribed dose $\left(\mathrm{V}_{95}\right)$ was $35.69 \pm 14.69 \mathrm{~cm}^{3}$ in the IMRT and $90.54 \pm 30.67 \mathrm{~cm}^{3}$ in the 3DCRT group, respectively $(p<0.001)$. In the IMRT group, the homogeneity index was $4.47 \pm 0.95$, and conformity index was $2.33 \pm 1.04$. In the subgroup analysis of T1a patients, volumes of carotid arteries that received at least $35 \mathrm{~Gy}\left(\mathrm{~V}_{35}\right)$ in the IMRT group was larger than that of the 3DCRT group: ipsilateral 
$\left(23.68 \pm 17.19 \mathrm{~cm}^{3}\right.$ vs. $\left.8.56 \pm 2.09 \mathrm{~cm}^{3} ; p<0.001\right)$, contralateral $\left(17.61 \pm 16.77 \mathrm{~cm}^{3}\right.$ vs. $\left.8.57 \pm 2.12 \mathrm{~cm}^{3} ; p<0.001\right)$. The doses to the organs at risk are presented in Table 2 .

\section{Treatment outcomes}

The local control rate of the 160 patients was $91.4 \%$ ( $\mathrm{T} 1 \mathrm{a}=$ $92.4 \%$ and $T 1 b=87.7 \% ; p=0.164)$ at 3 years. There was no significant difference in local control rate at 3 years between the IMRT and 3DCRT groups (94.4\% vs. 91.0\%; $p=0.587$ ) (Fig. 2). Fourteen of the 160 patients (8.75\%) had recurrences. The recurrences developed from 2 to 39 months after the completion of radiation therapy. Thirteen of 137 patients (9.5\%) in the 3DCRT group had recurrences. Of them, 10 patients had recurrences in the TVC only, 1 patient in the regional lymph nodes only, 1 patient in the adjacent thyroid and trachea with regional lymph nodes, and 1 patient in the lymph node and lung, respectively. Six of 13 recurring patients in the 3DCRT group had cordectomy, 4 total laryngectomy, 1 partial laryngectomy, and 1 chemotherapy.

Of a total of 23 IMRT patients, 1 (4.3\%) had a recurrence at the TVC. He had a partial laryngectomy with chemotherapy and did well with voice preservation for 38 months until the last follow-up date. The ultimate local control rate of the total of 160 patients was 100\% at 3 years. Details of recurrent patients are summarized in Table 3. The overall survival rates of the 160 patients were 100\%, 95.7\% at 3 and 5 years, respectively. There

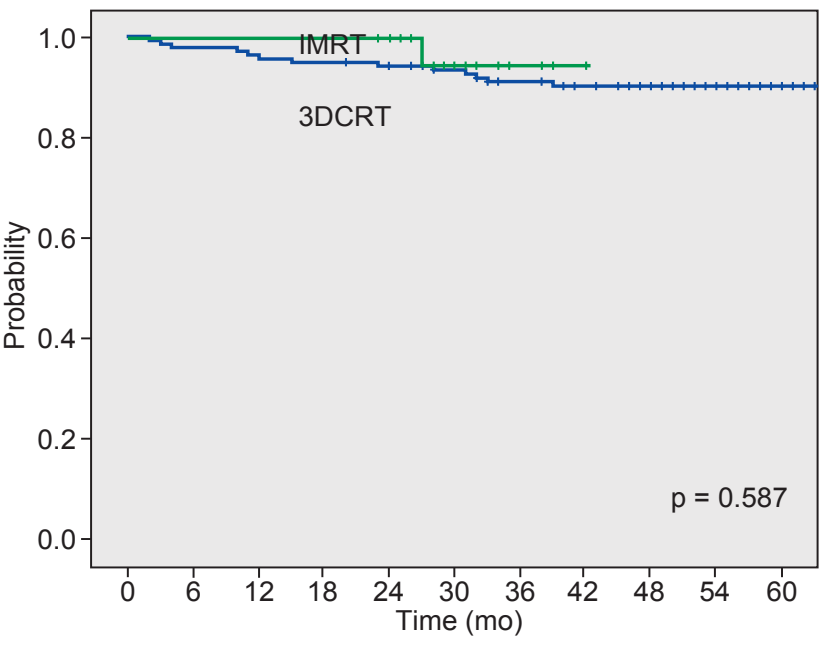

Fig. 2. Local recurrence-free survival rates by radiotherapy techniques (94.4\% of intensity-modulated radiotherapy [IMRT] vs. 91.0\% of three-dimensional conformal radiotherapy [3DCRT]; $p=$ 0.587).

was no significant difference in overall survival rates between the IMRT and 3DCRT groups at 3 years (100\% vs. 98.5\%; $p=$ 0.580). The disease-specific survival rates of the 160 patients were $100 \%$ and $99.9 \%$ at 3 and 5 years, respectively. There was no significant difference in disease-specific survival rates at 3 years between the IMRT and 3DCRT groups (100\% vs. 100\%; $p$ $=0.619$ ). All patients tolerated radiotherapy well.

Table 3. Details of recurred patients

\begin{tabular}{|c|c|c|c|c|c|c|c|c|c|c|}
\hline $\begin{array}{l}\text { Patient } \\
\text { No. }\end{array}$ & Age/Sex & $\begin{array}{c}\text { RT } \\
\text { technique }\end{array}$ & T-stage & $\begin{array}{l}\text { A-com } \\
\text { invasion }\end{array}$ & $\begin{array}{l}\text { LRFS } \\
\text { (mo) }\end{array}$ & Recur site & Treatment & $\begin{array}{c}\text { Voice } \\
\text { preservation }\end{array}$ & OS (mo) & Status \\
\hline 1 & $52 / \mathrm{M}$ & IMRT & $1 a$ & No & 27 & TVC & Partial L & Yes & 38 & Alive \\
\hline 2 & $64 / \mathrm{M}$ & 3DCRT & $1 a$ & Yes & 28 & LN & CTx & Yes & 64 & Alive \\
\hline 3 & $75 / \mathrm{M}$ & 3DCRT & $1 a$ & No & 12 & TVC & Cordectomy & Yes & 50 & Alive \\
\hline 4 & $72 / \mathrm{M}$ & 3DCRT & 16 & Yes & 32 & TVC & Cordectomy & Yes & 36 & Alive \\
\hline 5 & $69 / \mathrm{M}$ & 3DCRT & $1 a$ & No & 3 & TVC & Cordectomy & Yes & 35 & Alive \\
\hline 6 & $53 / \mathrm{M}$ & 3DCRT & 16 & Yes & 11 & TVC & Cordectomy & Yes & 74 & Alive \\
\hline 7 & $62 / \mathrm{M}$ & 3DCRT & $1 a$ & No & 23 & LN, DM & CTx & Yes & 29 & Alive \\
\hline 8 & $56 / \mathrm{M}$ & 3DCRT & $1 \mathrm{a}$ & No & 33 & TVC & Cordectomy & Yes & 109 & Alive \\
\hline 9 & $66 / \mathrm{M}$ & 3DCRT & 16 & Yes & 39 & TVC, DM & Total L, CTx & No & 54 & Died \\
\hline 10 & $64 / \mathrm{M}$ & 3DCRT & $1 a$ & No & 31 & TVC & Cordectomy & Yes & 95 & $A / D$ \\
\hline 11 & $66 / \mathrm{M}$ & 3DCRT & $1 a$ & No & 10 & TVC & Total L & No & 116 & Alive \\
\hline 12 & $69 / \mathrm{M}$ & 3DCRT & $1 a$ & No & 4 & TVC & Total L & No & 129 & Alive \\
\hline 13 & $68 / \mathrm{M}$ & 3DCRT & 16 & Yes & 2 & TVC & Total L & No & 142 & Alive \\
\hline 14 & $68 / \mathrm{M}$ & 3DCRT & 16 & Yes & 15 & TVC & Partial L & Yes & 117 & Alive \\
\hline
\end{tabular}

RT, radiotherapy; A-com, anterior commissure; LRFS, local recurrence-free survival; OS, overall survival; IMRT, intensity-modulated radiotherapy; 3DCRT, three-dimensional conformal radiotherapy; TVC, true vocal cord; LN, lymph node; DM, distant metastases; CTx, chemotherapy; L, laryngectomy; Died, died of lung metastases; A/D, alive with disease. 
Out of a total 137 3DCRT patients, acute skin reactions developed in the portal area during radiotherapy as follows: grade 1 in 108 (78.8\%) patients and grade 2 in 29 (21.2\%), respectively. Out of a total 23 IMRT patients, grade 1 skin reaction developed in 22 (95.6\%) patients and grade 2 in 1 (4.3\%) patient, respectively. The incidence of grade 2 (focal moist desquamation) was lower in the IMRT group (1/23, 4.3\%) than the 3DCRT group $(29 / 137,21.2 \%)$, but there was no statistical significance $(p=0.080)$. Hypothyroidism developed in one patient at 6 years, 8 months after 3DCRT. One patient had a tracheotomy because of dyspnea with severe radiation laryngitis that developed 8 months after the completion of 3DCRT.

\section{Discussion and Conclusion}

For patients with T1 or T2 glottic squamous cell cancer, surgery or radiotherapy may be used (3). Radiotherapy has been the primary treatment modality in early glottic cancer, with good tumor control and voice preservation. The use of 3DCRT with parallel opposing fields has been the standard technique. IMRT is as efficient as 3DCRT in terms of disease control and overall survival [11] and is a widely favored technique in the treatment of head and neck cancer [12-15]. The 5-year local control rate was reported as 94\% in T1a and 93\% in T1b glottic squamous cell carcinoma, respectively $[4,16]$. Zumsteg et al. [7] retrospectively analyzed $330 \mathrm{~T} 1$ and $\mathrm{T} 2$ patients and reported a 3-year local control rate of $88 \%$ with carotid-sparing IMRT and $89 \%$ with conventional RT, with no statistically significant differences. Berwouts et al. [11] reported a study that compared 40 patients with T1-T2 disease treated with IMRT without dose constraint for the carotid artery and 81 patients treated with conventional 3DCRT. There was no significant difference in 5-year local control between the groups, and significantly less skin toxicity in the IMRT group. Similarly, in our study there was no significant difference in the 3-year local control rate between the two techniques (94.4\% of IMRT vs. $91.0 \%$ of 3DCRT; $p=0.587$ ).

In the glottic cancer conventional dose-fractionation schedule, 1.8 Gy to 2.25 Gy per day, 5 days per week, has been used. Mendenhall and his colleagues $[3,4,17]$ favored fractionation usually exceeding 2 Gy per fraction, and often used 2.25 Gy per fraction for a total of 63 Gy in 28 fractions. There were some reports that hypofractionated radiation therapy resulted in comparable local control rates and toxicity compared with conventional fractionation [18-20].

The results of a prospective randomized study by the
Korean Radiation Oncology group suggested the potential advantages of using 2.25 Gy per fraction in terms of local control and shortened treatment time [19]. Al-Mamgani et al. [20] showed comparable local control and overall survival rates in T1a patients by hypofractionated single vocal cord irradiation with $58.08 \mathrm{~Gy}$ in 16 fractions. Traditionally, we favored 2 Gy per fraction rather than $2.25 \mathrm{~Gy}$; therefore, in this study, we included patients treated with $2 \mathrm{~Gy}$ per fraction for homogenous patient characteristics.

Cancer-specific survival rates over 95\% have been reported in early glottic cancer [4]. Therefore, it is of great concern that technical advances in radiotherapy can reduce longterm toxicity while maintaining good tumor control. IMRT significantly reduced unnecessary radiation doses to adjacent normal tissues, including the carotid arteries, compared with $3 \mathrm{D}$ conventional lateral fields $[4,21]$, so its use could be considered for some patients who have a high risk of potential stroke disease [21-23].

In contrast, there is controversy about using IMRT in the treatment of early glottic cancer because of a paucity of sensitive structure to spare with IMRT [5], and also the possibility of marginal missing of IMRT due to mis-targeting [4].

During deglutition, the movement of larynx can be up to $3.5 \mathrm{~cm}$ in the superior-inferior direction [24]. Vocal cord motion during regular breathing should be accounted for when treatment volumes are significantly reduced [20]. Vocal cord displacement can be more accurately studied with fourdimensional CT scanning and IGRT, and PTV margin might be further reduced $[9,25]$. Gujral et al. [9] reviewed and summarized 16 references on carotid sparing IMRT for early glottis cancer and found that adequate allowance for laryngeal movement during swallowing and breathing is crucial in determining a PTV that balances vocal cord displacement and sparing the carotid arteries. The use of magnetic resonance imaging for radiotherapy planning may improve tumor localization and quantification of motion during treatment [26]. For dose to the carotid PRV volumes, proton planning had superiority compared with 3D planning [27].

In our hospital, LMS biopsy and stripping is performed before radiotherapy. Therefore, gross lesions are not visible. $\mathrm{T} 1$ lesions of glottic cancer have a low risk of microscopic spread to cartilaginous structure [9]. We focused radiotherapy planning to minimize the treated volume of adjacent healthy laryngeal tissue when we began the IMRT technique for T1 glottic cancer. To cover potential microscopic spread, we defined the planning target volume of the high-risk area (named PTV-2) including adjacent supra- and subglottic tissues 
that prescribed 59.4 Gy in 33 fractions. In this study, $V_{95}$ (treated volume of 95\% prescribed dose) was lower in the IMRT group than the 3DCRT group. For the organs at risk we delineated thyroid glands, esophagus and spinal cord. The carotid arteries were not routinely delineated. We retrospectively defined the PRV of carotid artery for T1a patients following the recommendation of the literature [9]. The 2 patients in the IMRT group who were treated with carotid artery dose constraint were excluded from the dose analysis. Because of the no dose constraint to the carotid artery, the volume of the ipsilateral carotid artery that received at least 35 Gy $\left(V_{35}\right)$ was higher in the IMRT group than that in the 3DCRT group, while $V_{50}$ was lower in the IMRT group.

Berwouts et al. [11] reported less incidence and severity of acute dermatitis during IMRT than 3DCRT. In our study, the incidence of grade 2 acute radiation dermatitis was less in patients who received IMRT than 3DCRT (4.3\% vs. 21.2\%; $p=0.080$ ), but had no statistical significance. Patients who underwent IMRT received more mean doses in the thyroid gland and maximum point doses in the spinal cord than the 3DCRT group. However, the doses delivered with the two techniques were under the limited value of normal tissue tolerance.

This study has several limitations as a retrospective analysis. The follow-up duration of patients treated with IMRT (median of 30 months) is short for survival analysis. The sample size of the IMRT group was also too small for statistics. National Health Insurance has provided benefits coverage for IMRT since 2015, and from that time, IMRT has been routinely applied to head-and-neck cancer patients in our hospital. In the future, further evaluation will be necessary with a larger patient population and longer follow-up period.

In conclusion, IMRT had a comparable local control rate and radiation toxicity compared with 3DCRT in stage 1 glottic cancer patients.

\section{Conflict of Interest}

No potential conflict of interest relevant to this article was reported.

\section{References}

1. Mo HL, Li J, Yang $X$, et al. Transoral laser microsurgery versus radiotherapy for T1 glottic carcinoma: a systematic review and meta-analysis. Lasers Med Sci 2017;32:461-7.

2. Taylor SM, Kerr P, Fung K, et al. Treatment of T1b glottic SCC: laser vs. radiation: a Canadian multicenter study. J Otolaryngol Head Neck Surg 2013;42:22.

3. Mendenhall WM, Werning JW, Hinerman RW, Amdur RJ, Villaret DB. Management of T1-T2 glottic carcinomas. Cancer 2004;100:1786-92.

4. Chera BS, Amdur RJ, Morris CG, Kirwan JM, Mendenhall WM. T1NO to T2NO squamous cell carcinoma of the glottic larynx treated with definitive radiotherapy. Int J Radiat Oncol Biol Phys 2010;78:461-6.

5. Mourad WF, Hu KS, Shourbaji RA, Woode R, Harrison LB. Long-term follow-up and pattern of failure for T1-T2 glottic cancer after definitive radiation therapy. Am J Clin Oncol 2013;36:580-3.

6. Khaja SF, Hoffman HT, Pagedar NA. Treatment and survival trends in glottic carcinoma in situ and stage I cancer from 1988 to 2012. Ann Otol Rhinol Laryngol 2016;125:311-6.

7. Zumsteg ZS, Riaz N, Jaffery S, et al. Carotid sparing intensity-modulated radiation therapy achieves comparable locoregional control to conventional radiotherapy in T1-2NO laryngeal carcinoma. Oral Oncol 2015;51:716-23.

8. Cox JD, Stetz J, Pajak TF. Toxicity criteria of the Radiation Therapy Oncology Group (RTOG) and the European Organization for Research and Treatment of Cancer (EORTC). Int J Radiat Oncol Biol Phys 1995;31:1341-6.

9. Gujral DM, Long M, Roe JW, Harrington KJ, Nutting CM. Standardisation of target volume delineation for carotidsparing intensity-modulated radiotherapy in early glottis cancer. Clin Oncol (R Coll Radiol) 2017;29:42-50.

10. Special considerations regarding absorbed-dose and dosevolume prescribing and reporting in IMRT. J ICRU 2010;10:2740.

11. Berwouts D, Swimberghe M, Duprez F, et al. Intensitymodulated radiotherapy for early-stage glottic cancer. Head Neck 2016;38 Suppl 1:E179-84.

12. Kwong DL, Pow EH, Sham JS, et al. Intensity-modulated radiotherapy for early-stage nasopharyngeal carcinoma: a prospective study on disease control and preservation of salivary function. Cancer 2004;101:1584-93.

13. Saarilahti K, Kouri M, Collan J, et al. Intensity modulated radiotherapy for head and neck cancer: evidence for preserved salivary gland function. Radiother Oncol 2005;74:251-8.

14. Broglie MA, Soltermann A, Haile SR, et al. Quality of life of oropharyngeal cancer patients with respect to treatment strategy and p16-positivity. Laryngoscope 2013;123:164-70.

15. Kerr P, Myers CL, Butler J, Alessa M, Lambert P, Cooke AL. Prospective functional outcomes in sequential population based cohorts of stage III/IV oropharyngeal carcinoma 
patients treated with 3D conformal vs. intensity modulated radiotherapy. J Otolaryngol Head Neck Surg 2015;44:17.

16. Greulich MT, Parker NP, Lee P, Merati AL, Misono S. Voice outcomes following radiation versus laser microsurgery for T1 glottic carcinoma: systematic review and meta-analysis. Otolaryngol Head Neck Surg 2015;152:811-9.

17. Mendenhall WM, Amdur RJ, Morris CG, Hinerman RW. T1T2N0 squamous cell carcinoma of the glottic larynx treated with radiation therapy. J Clin Oncol 2001;19:4029-36.

18. Gowda RV, Henk JM, Mais KL, Sykes AJ, Swindell R, Slevin NJ. Three weeks radiotherapy for $\mathrm{T} 1$ glottic cancer: the Christie and Royal Marsden Hospital Experience. Radiother Oncol 2003;68:105-11.

19. Moon SH, Cho KH, Chung EJ, et al. A prospective randomized trial comparing hypofractionation with conventional fractionation radiotherapy for T1-2 glottic squamous cell carcinomas: results of a Korean Radiation Oncology Group (KROG-0201) study. Radiother Oncol 2014;110:98-103.

20. Al-Mamgani A, Kwa SL, Tans L, et al. Single vocal cord irradiation: image guided intensity modulated hypofractionated radiation therapy for T1a glottic cancer: early clinical results. Int J Radiat Oncol Biol Phys 2015;93:33743.
21. Rosenthal DI, Fuller CD, Barker JL Jr, et al. Simple carotidsparing intensity-modulated radiotherapy technique and preliminary experience for T1-2 glottic cancer. Int J Radiat Oncol Biol Phys 2010;77:455-61.

22. Wilbers J, Dorresteijn LD, Haast R, et al. Progression of carotid intima media thickness after radiotherapy: a long-term prospective cohort study. Radiother Oncol 2014;113:359-63.

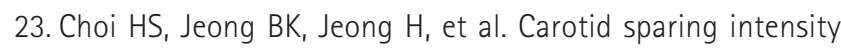
modulated radiotherapy on early glottic cancer: preliminary study. Radiat Oncol J 2016;34:26-33.

24. Molfenter SM, Steele CM. Physiological variability in the deglutition literature: hyoid and laryngeal kinematics. Dysphagia 2011;26:67-74.

25. Osman SO, Astreinidou E, de Boer HC, et al. IMRT for imageguided single vocal cord irradiation. Int J Radiat Oncol Biol Phys 2012;82:989-97.

26. Schmidt MA, Payne GS. Radiotherapy planning using MRI. Phys Med Biol 2015;60:R323-61.

27. Matthiesen $C$, de la Fuente Herman $T$, Singh $H_{\text {, et al. Dosimetric }}$ and radiobiologic comparison of $3 \mathrm{D}$ conformal, IMRT, VMAT and proton therapy for the treatment of early-stage glottic cancer. J Med Imaging Radiat Oncol 2015;59:221-8. 